\title{
CONCENTRATION OF THE DISTANCE IN FINITE DIMENSIONAL NORMED SPACES
}

\author{
JuAN ARIAS-DE-ReynA ${ }^{1}$ \\ KEITH BALL ${ }^{2}$ \\ RAFAEL VILLA ${ }^{1}$
}

\begin{abstract}
We prove that in every finite dimensional normed space, for "most" pairs $(x, y)$ of points in the unit ball, $\|x-y\|$ is more than $\sqrt{2}(1-\varepsilon)$. As a consecuence, we obtain a result proved by Bourgain, using QS-descomposition, that guarantees an exponentially large number of points in the unit ball any two of which are separated by more than $\sqrt{2}(1-\varepsilon)$.
\end{abstract}

\section{Introduction and previous results.}

Let $X=\left(\mathbb{R}^{n},\|\cdots\|\right)$ be an $n$-dimensional normed space, $K=\left\{x \in \mathbb{R}^{n}:\|x\| \leq 1\right\}$ its closed unit ball and let $m$ be the Lebesgue measure restricted to $K$, normalized so that $m(K)=1$. It easy to see that when $n$ is large, most of the points in $K$ lie near its surface: so their norm is about 1 . In this article, our aim is to investigate the typical behaviour of the distance between two points in the ball.

The investigation is motivated by a number of recent results showing that in a wide variety of special spaces, it is possible to find many points, any two of which are roughly distance 1 apart. Given $X$ of dimension $n$, let $N=N(\varepsilon)$ be the highest cardinality of a subset $T$ of $X$ such that

$$
1-\varepsilon \leq\|x-y\| \leq 1+\varepsilon, \quad \text { for all } x, y \in T, x \neq y \text {. }
$$

A volume argument easily shows that $N(\varepsilon) \leq \exp \{\varphi(\varepsilon) n\}$ for some function $\varphi$, independent of $X$. In $[\mathrm{BBK}]$ an exponential estimate from below is obtained, for finite dimensional spaces with a 1-subsymmetric basis: in particular, for example, for $\ell_{p}$ spaces. Some extensions of this result appear in [BB], where sharp estimates are given for the case of the space $\ell_{p}^{n}\left(\ell_{q}^{m}\right)(1 \leq p, q<\infty)$. Related results can be found in [BPS1], [BPS2] and [BMW].

1991 Mathematics Subject Classification. 46B04, 46E15.

Key words and phrases. Normed space, almost equidistant points, concentration of distance, concentration of measure, uniformly convex spaces.

${ }^{1}$ Research supported by DGICYT grant \#PB93-0926.

${ }^{2}$ Research supported in part by NSF DMS-9257020.

This paper includes part of the Ph. D. thesis of the third author.

Typeset by $\mathcal{A M}_{\mathcal{S}}-\mathrm{T}_{\mathrm{EX}}$ 
The results in each of these articles depend upon the construction of some probability measure on the unit ball with respect to which, two independent random vectors are almost exactly a fixed distance apart with very high probability. In this article we consider the simplest such measure, Lebesgue measure, and examine the distribution of the random variable $\|x-y\|(x, y \in K)$,

$$
F(t)=m \otimes m\{(x, y) \in K \times K:\|x-y\| \leq t\} .
$$

If $F(t)$ jumps from $\delta$ to $1-\delta$ as $t$ goes from $a(1-\varepsilon)$ to $a(1+\varepsilon)$, then we can find $N$ points $x_{1}, \ldots, x_{N} \in K$, such that $a^{-1} x_{1}, \ldots, a^{-1} x_{N}$ satisfy (1), as long as $2 N^{2} \delta \leq 1$. Our interest is in exponentially large sets of points so we shall look for a number $a$ for which we have something like

$$
F(a(1+\varepsilon))-F(a(1-\varepsilon))>1-\exp \left\{-2 c \varepsilon^{2} n\right\}
$$

for some constant $c$.

In Section 1, we show that there are normed spaces for which there is no threshold behaviour for the distribution function. However, our main result guarantees that in all normed spaces, the distance between "most" pairs of points in the unit ball is greater than $\sqrt{2}(1-\varepsilon)$. We show, in fact, that for any space,

$$
F(\sqrt{2}(1-\varepsilon))<\exp \left(\frac{-n \varepsilon^{2}(2-\varepsilon)^{2}}{2}\right) .
$$

Hence, in particular, if the distance does concentrate around a number, then this number must be no smaller than $\sqrt{2}$. This result is sharp in that, for Euclidean space, there is a threshold at $\sqrt{2}$. (In this case

$$
F(\sqrt{2}(1-\varepsilon))>\frac{1}{2 \sqrt{n}}\left(1-\varepsilon^{2}(2-\varepsilon)^{2}\right)^{\frac{n}{2}},
$$

so our argument actually recovers the "correct" exponent.)

Section 2 is concerned with spaces which do exhibit some kind of threshold behaviour. It is shown in $[\mathrm{GM}]$ (see $[\mathrm{A}]$ for an easier proof along the same lines) that the balls of uniformly convex spaces enjoy a concentration of measure phenomenon: if $K$ is such a set, then for any $\varepsilon>0$ and any Borel set $A \subset K$ with $m(A) \geq 1 / 2$, the measure of the expanded set $A_{\varepsilon}=\{x \in K: \operatorname{dist}(x, A) \leq \varepsilon\}$ satisfies

$$
m\left(A_{\varepsilon}\right) \geq 1-e^{-\varphi(\varepsilon) n}
$$

for some positive function $\varphi$. We have included a very short proof of this fact. We then observe that such a concentration of measure guarantees threshold behaviour for our distribution $F$.

We should mention that there are some results known for isometric embeddings, see $[\mathrm{P}]$, and that infinite-dimensional analogues of some of these problems appear in $[\mathrm{D}]$ and $[\mathrm{EO}]$.

\section{$\S 1$. Large distances.}

As above, let $X=\left(\mathbb{R}^{n},\|\cdots\|\right)$ be an $n$-dimensional normed space, let $K=\left\{x \in \mathbb{R}^{n}:\|x\| \leq 1\right\}$ be its closed unit ball and let $m$ be the Lebesgue measure on $K$ normalized so that $m(K)=1$. The 
distribution function of the distance between two independent points, each distributed uniformly on $K$, is given by,

$$
F(t)=m \otimes m\{(x, y) \in K \times K:\|x-y\| \leq t\} .
$$

It is easy to check that

$$
F(t)=\int_{t K} m(K \cap(x+K)) d m(x) .
$$

We begin the section with some simple examples showing, among other things, that there are spaces for which $F$ does not have a sharp threshold.

EXAmples. (1) $X=\ell_{2}^{n}$. It is clear from standard results that the Euclidean distance between independent random points in the Euclidean ball, concentrates around $\sqrt{2}$ since both points are very close to the surface and each one is almost certain to be near the equator "perpendicular to" the other. However, it will be useful later, if we examine the distribution more carefully.

For any $x \in 2 K$,

$$
m(K \cap(x+K))=\frac{2}{v_{n}} v_{n-1} \int_{r / 2}^{1}\left(1-s^{2}\right)^{\frac{n-1}{2}} d s,
$$

where $r=\|x\|$ and $v_{n}$ is the volume of the unit ball in $\ell_{2}^{n}$. Integrating over $t K$ we get

$$
F(t)=\frac{2 n v_{n-1}}{v_{n}} \int_{0}^{t} r^{n-1}\left(\int_{r / 2}^{1}\left(1-s^{2}\right)^{\frac{n-1}{2}} d s\right) d r
$$

From this we get that the density of the random variable $\|x-y\|$ is roughly

$$
t \mapsto \sqrt{\frac{n}{\pi}}\left(\frac{t^{2}\left(4-t^{2}\right)}{4}\right)^{\frac{n}{2}} .
$$

(A similar expression will reappear later on.)

(2) $X=\ell_{\infty}^{n}$. For $0 \leq t \leq 2$,

$$
F(t)=\int_{\mathbb{R}^{2 n}} \chi_{K}(x) \chi_{K}(y) \chi_{t K}(x-y) d m(x) d m(y)
$$

is $n^{\text {th }}$ power of the two-dimensional measure of the set

$$
\left\{(x, y) \in \mathbb{R}^{2}:|x| \leq 1,|y| \leq 1,|x-y| \leq t\right\},
$$

and so,

$$
F(t)=\left(\frac{t(4-t)}{4}\right)^{n}
$$


Since $t(4-t)$ attains its maximum at $t=2$, this shows that the distance in the ball of $\ell_{\infty}^{n}$ concentrates around $a=2$.

(3) The next example shows that there are normed spaces where concentration of the distance does not occur. The real point is that concentration can occur at different points for different spaces. Let $X=\left(\ell_{2}^{n} \oplus \mathbb{R}\right)_{\infty}=\left(\mathbb{R}^{n+1},\|\cdots\|\right)$, where

$$
\|(x, y)\|=\sup \left\{\|x\|_{2},|y|\right\}, \quad x \in \ell_{2}^{n}, y \in \mathbb{R} .
$$

Thus $K$ is a "circular" cylinder in $\mathbb{R}^{n+1}$.

It is easy to check that for a Cartesian product set, the distribution function $F$ is just the product of the distributions for the two factors. So for our space, $F(t)=F_{1}(t) F_{2}(t), F_{1}$ being the distribution function for $\ell_{2}^{n}$ and $F_{2}$ being the one for $\mathbb{R}$. $F_{1}$ has a sharp threshold at $\sqrt{2}$ and so is almost equal to 1 on the interval $[\sqrt{2}(1+\varepsilon), 2]$. Thus, $F$ is almost the same as $F_{2}$ from $\sqrt{2}$ onwards. From example (2) we see that $F_{2}$ is given by

$$
F_{2}(t)=\frac{t(4-t)}{4}
$$

and hence $F$ has no sharp threshold.

Despite examples like the preceding one, our main result, Theorem 1 below, shows that in any normed space "most" of the pairs of points in the unit ball are separated by more than $\sqrt{2}(1-\varepsilon)$ : in particular, that we may find exponentially many, that are so separated. This fact was proved by Bourgain using QS-descomposition (see [FL] for a proof). It was proved in [EO] that in any infinite-dimensional normed space, there is an infinite subset of the unit ball whose elements are $(1+\varepsilon)$-separated, for some $\varepsilon>0$ depending on the normed space. Theorem 1 seems stronger than this, in spirit, but in $[\mathrm{BRR}]$ it was shown that the greatest separation of any infinite subset in the unit ball of $\ell_{p}(1 \leq p<\infty)$ is $2^{1 / p}$ : so Theorem 1 cannot be used to improve the infinite dimensional statement.

Theorem 1. Let $X$ be an $n$-dimensional normed space, $K$ its closed unit ball and $m$, the Lebesgue measure on $K$ normalized so that $m(K)=1$. Then, for any $0<t<\sqrt{2}$

$$
m \otimes m\{(x, y) \in K \times K:\|x-y\| \leq t\} \leq\left(\frac{t^{2}\left(4-t^{2}\right)}{4}\right)^{\frac{n}{2}} .
$$

If $t=\sqrt{2}(1-\varepsilon)$ the latter is no more than

$$
\exp \left\{-\varepsilon^{2} n / 2\right\} \text {. }
$$

Note. The sharpness of the result can be gauged by referring to the example $X=\ell_{2}^{n}$ discussed earlier. 
Proof. For any $t \in[0,2]$, let

$$
F(t)=m \otimes m\{(x, y) \in K \times K:\|x-y\| \leq t\} .
$$

It is clear that $F(t)$ is a value of the threefold convolution of the characteristic functions of $K$ (twice) and $t K$,

$$
F(t)=\chi_{t K} * \chi_{K} * \chi_{K}(0) .
$$

To estimate this we use the sharp form of Young's inequality for convolutions on $\mathbb{R}^{n}$ (see note below): for any $p, q, r \geq 1$ with $\frac{1}{p}+\frac{1}{q}+\frac{1}{r}=2$ there is a constant $C(p, q, r ; n)>0$ such that for any functions $f \in L^{p}\left(\mathbb{R}^{n}\right), g \in L^{q}\left(\mathbb{R}^{n}\right)$ and $h \in L^{r}\left(\mathbb{R}^{n}\right)$, we have

$$
|f * g * h(0)| \leq C(p, q, r ; n)\|f\|_{p}\|g\|_{q}\|h\|_{r} .
$$

The sharp constant $C(p, q, r ; n)$ equals $\left(C_{p} C_{q} C_{r}\right)^{n}$, where $C_{p}^{2}=p^{1 / p}{p^{\prime}}^{-1 / p^{\prime}}$ ( $p^{\prime}$ being the conjugate exponent to $p$ ). If $q=r$ we obtain

$$
C(p, q, q ; n)=\left[\frac{(2 q)^{1 / q}}{2}\left(\frac{2}{q}-1\right)^{1 / q-1 / 2}\right]^{n}
$$

for any $p, q \geq 1$ with $\frac{1}{p}+\frac{2}{q}=2$. Hence, for any $q \geq 1$,

$$
\begin{aligned}
F(t) & =\chi_{t K} * \chi_{K} * \chi_{K}(0) \leq\left[\frac{(2 q)^{1 / q}}{2}\left(\frac{2}{q}-1\right)^{1 / q-1 / 2}\right]^{n}\left\|\chi_{t K}\right\|_{p}\left\|\chi_{K}\right\|_{q}^{2} \\
& =\left[t^{2(1-1 / q)} \frac{(2 q)^{1 / q}}{2}\left(\frac{2}{q}-1\right)^{1 / q-1 / 2}\right]^{n} .
\end{aligned}
$$

For $t<\sqrt{2}$, the last expression is minimum for $q=\frac{4-t^{2}}{2}$ and for this $q$ the expression simplies to give

$$
F(t) \leq\left(\frac{t^{2}\left(4-t^{2}\right)}{4}\right)^{n / 2}
$$

Then, if $0<\varepsilon<1$, we have

$$
\begin{aligned}
F(\sqrt{2}(1-\varepsilon)) & \leq\left(\frac{(\sqrt{2}(1-\varepsilon))^{2}\left(4-(\sqrt{2}(1-\varepsilon))^{2}\right)}{4}\right)^{n / 2}=\left(1-\varepsilon^{2}(2-\varepsilon)^{2}\right)^{n / 2} \\
& \leq \exp \left\{-\varepsilon^{2} n / 2\right\} .
\end{aligned}
$$

NotE. The sharp constant in Young's inequality was determined by Beckner, [Be], in connection with his study of the Fourier transform. An important extension of Young's inequality, again with sharp constant, was proved by Brascamp and Lieb, [BL]. Recently, a very elegant new proof of their inequality was found by F. Barthe [Ba]. 
$\S 2$. A concentration of measure phenomenon. Uniformly convex spaces. We begin this section with a short proof of the concentration phenomenon in uniformly convex spaces, proved by Gromov and Milman, [GM]. The origin of our argument is in a paper of Talagrand [T] concerning Gauss space. A simplification of his argument was found by Maurey $[\mathrm{M}]$, and was used by Schmuckensläger [S] to study uniformly convex spaces. The proof below is in the same spirit. Unfortunately, the proof is now so short that one can't see the ideas behind it.

For a uniformly convex normed space $X$, we define the modulus of convexity, $\delta$, of $X$ by

$$
\delta(\varepsilon)=\inf \left\{1-\left\|\frac{x+y}{2}\right\|:\|x-y\| \geq \varepsilon,\|x\| \leq 1,\|y\| \leq 1\right\} .
$$

This definition is slightly different from that in the standard texts but the difference is of no real consequence. As before, for a subset $A$ of $X$ and a number $\varepsilon>0$ we write $A_{\varepsilon}$ for the set of points whose distance (in norm) from $A$ is at most $\varepsilon$.

Theorem 2. Let $X$ be an n-dimensional uniformly convex normed space, $K$ its closed unit ball, $m$ the normalized Lebesgue measure on $K$ and let $\delta$ be the modulus of convexity of $X$. Then for any Borel set $A \subset K$ with $m(A) \geq 1 / 2$ and any $\varepsilon>0$,

$$
m\left(A_{\varepsilon}\right)>1-2 e^{-2 n \delta(\varepsilon)} .
$$

Proof. Recall the multiplicative Brunn-Minkowski inequality:

$$
m\left(\frac{A+B}{2}\right) \geq m(A)^{1 / 2} m(B)^{1 / 2} .
$$

Suppose $A \subset K$ and put

$$
B=\{y \in K: d(y, A) \geq \varepsilon\} .
$$

If $x \in A$ and $y \in B$ then $\|x-y\| \geq \varepsilon$ and hence

$$
\left\|\frac{x+y}{2}\right\| \leq 1-\delta(\varepsilon) .
$$

Therefore

$$
\frac{A+B}{2} \subset(1-\delta(\varepsilon)) K
$$

and so

$$
m(A) m(B) \leq(1-\delta(\varepsilon))^{2 n} \leq e^{-2 n \delta(\varepsilon)} .
$$

The remainder of this section is devoted to showing that a concentration of measure such as the above, suffices to guarantee that the distance between two independent points in the unit ball, concentrates around some number. 
Theorem 3. Let $X$ be an $n$-dimensional normed space, $K$ its closed unit ball and $m$ the normalized Lebesgue measure on $K$. Assume that there exists an increasing function $\varphi$ such that for any $\varepsilon>0$ and any Borel set $A \subset K$ satisfying $m(A) \geq 1 / 2$,

$$
m\left(A_{\varepsilon}\right) \geq 1-e^{-\varphi(\varepsilon) n} .
$$

Then there exists a number $a \in[1 / 2,2]$ such that for any $\varepsilon>0$,

$$
m \otimes m\{(x, y) \in K \times K: a(1-\varepsilon) \leq\|x-y\| \leq a(1+\varepsilon)\} \geq 1-4 \exp \{-\varphi(\varepsilon / 6) n\},
$$

and hence, for any $\varepsilon>0$ there exist $N$ points $x_{1}, \ldots, x_{N} \in X$ so that

$$
1-\varepsilon \leq\left\|x_{i}-x_{j}\right\| \leq 1+\varepsilon \quad 1 \leq i<j \leq N,
$$

as long as $N<(1 / 2) \exp \{\varphi(\varepsilon / 6) n / 2\}$.

Proof. For each $x \in K$, consider the function $f_{x}: K \rightarrow \mathbb{R}$ defined by $f_{x}(y)=\|x-y\|$. Let $\alpha(x)$ be the median of $f_{x}$ so that the ball of radius $\alpha(x)$ includes half of $K$. (The median is clearly unique.) Whatever the dimension, $\frac{1}{2} K$ has volume at most one half that of $K$ so $\alpha(x)$ is at least $\frac{1}{2}$ for each $x$.

Let $a$ be a median of the function $\alpha$. We have $a \geq 1 / 2$.

Now let $A=\{x \in K: \alpha(x) \leq a\}$. Since $A_{a \varepsilon} \subset\{x \in K: \alpha(x) \leq a(1+\varepsilon)\}$, the concentration hypothesis ensures that,

$$
m(\{x \in K: \alpha(x) \leq a(1+\varepsilon)\}) \geq 1-\exp \{-\varphi(a \varepsilon) n\} \geq 1-\exp \{-\varphi(\varepsilon / 2) n\} .
$$

Similarly

$$
m(\{x \in K: \alpha(x) \geq a(1-\varepsilon)\}) \geq 1-\exp \{-\varphi(\varepsilon / 2) n\},
$$

and hence

$$
m(\{x \in K: a(1-\varepsilon) \leq \alpha(x) \leq a(1+\varepsilon)\}) \geq 1-2 \exp \{-\varphi(\varepsilon / 2) n\} .
$$

The same argument can be applied for each fixed $x \in K$ to get

$$
m(\{y \in K: \alpha(x)(1-\varepsilon) \leq\|x-y\| \leq \alpha(x)(1+\varepsilon)\}) \geq 1-2 \exp \{-\varphi(\varepsilon / 2) n\} .
$$

These two inequalities give

$$
m \otimes m\left(\left\{(x, y) \in K \times K: a(1-\varepsilon)^{2} \leq\|x-y\| \leq a(1+\varepsilon)^{2}\right\}\right) \geq 1-4 \exp \{-\varphi(\varepsilon / 2) n\},
$$

and thus, for any $\varepsilon<1$

$$
m \otimes m(\{(x, y) \in K \times K: a(1-3 \varepsilon) \leq\|x-y\| \leq a(1+3 \varepsilon)\}) \geq 1-4 \exp \{-\varphi(\varepsilon / 2) n\} .
$$

So

$$
m \otimes m(\{(x, y) \in K \times K: a(1-\varepsilon) \leq\|x-y\| \leq a(1+\varepsilon)\}) \geq 1-4 \exp \{-\varphi(\varepsilon / 6) n\},
$$

for every $\varepsilon>0$, as required.

The conclusion concerning the choice of $N$ points is now obvious. 


\section{REFERENCES}

[A] S. Alesker, On the Gromov-Milman theorem regarding the concentration phenomenon on uniformly convex sphere, to appear.

[Ba] F. Barthe, Geometric and Functional Analysis (to appear).

[BB] J. Bastero, J. Bernués, Applications of deviation inequalities on finite metric sets, Math. Nachr. 153 (1991), 33-41.

[BBK] J. Bastero, J. Bernués, N. Kalton, Embedding $\ell_{\infty}^{n}$-cubes in finite dimensional 1-subsymmetric spaces, Rev. Mat. Univ. Complut. Madrid 2 (1989), 47-52.

[BPS1] J. Bastero, A. Peña, G. Schechtman, Embeddings $\ell_{\infty}^{n}$-cubes in the orbit of an element in commutative and noncommutative $\ell_{p}^{n}$-spaces, Seminario Dpto. Análisis Matemático, Univ. Complut. Madrid.

[BPS2] J. Bastero, A. Peña, G. Schechtman, Embeddings $\ell_{\infty}^{n}$-cubes in low dimensional Schatten classes, Geometric Aspects of Functional Analysis (Israel, 1992-1994) (1995), 5-11.

[Be] W. Beckner, Inequalities in Fourier analysis, Ann. of Math. 102 (1975), 159-182.

[BMW] J. Bourgain, V. Milman, H. Wolfson, On type of metric spaces, Trans. Amer. Math. Soc. 294 (1986), 295-317.

[BL] H. J. Brascamp and E. H. Lieb, Best constants in Young's inequality, its converse, and its generalization to more than three functions, Advances in Math. 20 (1976), 151-173.

[BRR] J. A. C. Burlak, R. A. Rankin and A. P. Robertson, The packing of spheres in the space $\ell_{p}$, Proc. Glasgow Math. Assoc. 4 (1958), 22-25.

[D] T. Domínguez-Benavides, Some properties of the set and ball measures of noncompactness and applications, J. London Math. Soc. (2) 34 (1986), 120-128.

[EO] J. Elton and E. Odell, The unit ball of every infinite-dimensional normed linear space contains a $(1+\varepsilon)-$ separated sequence, Colloq. Math. 44 (1981), 105-109.

[FL] Z. Füredi and P. A. Loeb, On the best constant for the Besicovitch covering theorem, Proc. Amer. Math. Soc. 121 (1994), 1063-1073.

[GM] M. Gromov and V. D. Milman, Generalization of the spherical isoperimetric inequality to uniformly convex Banach spaces, Compositio Math. 62 (1987), 263-282.

[K] C. A. Kottman, Subsets of the unit ball that are separated by more than one, Studia Math. 53 (1975), $15-27$.

[M] B. Maurey, Some deviation inequalities, Geom. Funct. Anal. 1 (1991), 188-197.

[Pe] C. M. Petty, Equilateral sets in Minkowski spaces, Proc. Amer. Math. Soc. 29 (1971), 369-374. 
[S] M. Schmuckenschläger, A concentration of measure phenomenon on uniformly convex bodies, Geometric Aspects of Functional Analysis (Israel, 1992-1994). (1995), 275-287.

[T] M. Talagrand, A new isoperimetric inequality and the concentration of measure phenomenon, Geometric Aspects of Functional Analysis (Israel Seminar, 1989-1990). Lecture Notes in Math 1469 (1991), 94-124.

Keith M. BAll address: Department of Mathematics, University College London, Gower Street, LONDON WC1E 6BT. ENGLAND.

E-mail address: kmb@math.ucl.ac.uk

JUAN ARIAS-DE-REYNA address: Dpto. Análisis Matemático, Facultad de Matemáticas, UniverSidAd de Sevilla, C/ TARfia, s/n, 41012 SEVILLA. SPAin.

E-mail address: arias@cica.es

RAFAel Villa address: Dpto. Análisis Matemático, Facultad de Matemáticas, Universidad de Sevilla, c/ Tarfia, s/n, 41012 SEVILlA. SPAIN.

E-mail address: rvcaro@cica.es 